\title{
Effects of self-consistency violation in Hartree-Fock RPA calculations for nuclear giant resonances revisited
}

\author{
Tapas Sil ${ }^{1}$, S. Shlomo ${ }^{1}$, B. K. Agrawal ${ }^{1,2}$, and P.-G. Reinhard ${ }^{3}$ \\ 1 Cyclotron Institute, Texas A\&M University, \\ College Station, Texas 7ry 4 3, USA \\ 2 Saha Institute of Nuclear Physics, \\ 1/AF Bidhannagar, Kolkata 700064, India \\ 3 Institut für Theoretische Physik, Universität Erlangen, D-91058 Erlangen, Germany,
}

(Dated: August 31, 2018)

\begin{abstract}
We provide accurate assessments of the consequences of violations of self-consistency in HartreeFock (HF) based random phase approximation (RPA) calculations of the centroid energy $E_{c e n}$ of isoscalar and isovector giant resonances of multi-polarities $L=0-3$ in a wide range of nuclei. This is done by carrying out highly accurate HF-RPA calculations neglecting the particle-hole (ph) spin-orbit or Coulomb interaction in the RPA and comparing with the fully self-consistent HF-RPA results. We find that the shifts in the value of $E_{c e n}$ due to self-consistency violation associated with the spin-orbit and Coulomb interactions are comparable or larger than the current experimental errors in $E_{c e n}$.
\end{abstract}

PACS numbers: 24.30.Cz, 21.65.+f, 21.60.Jz 


\section{INTRODUCTION}

The study of collective modes in nuclei provide very important information for understanding the structural and bulk properties of nuclear systems. For example, the isovector giant dipole (IVGDR) mode is sensitive to the symmetry energy, the centroid energy $E_{c e n}$ of the isoscalar giant monopole resonance (ISGMR) is related to the value of the incompressibility modulus $K_{\infty}$ of symmetric nuclear matter [1, 2], and low lying collective states give a clue on the nuclear shell structure. These quantities are important ingredients not only for the description of finite nuclei but also for the study of heavy-ion collisions, supernovae and neutron stars. Recent developments in high precision experimental facilities make it possible to measure the centroid energy of the ISGMR with an error of $\delta E_{c e n} \sim 0.1-0.3$ $\mathrm{MeV}$ 3, 4]. Using the approximate relation $\left(\delta K_{\infty}\right) / K_{\infty}=2\left(\delta E_{c e n}\right) / E_{c e n}$ and, for example, the recent experimental value of $E_{c e n}=13.96 \pm 0.20 \mathrm{MeV}$ for the ISGMR in ${ }^{208} \mathrm{~Pb}$, one has an error of $\delta K_{\infty}=6-9 \mathrm{MeV}$ for $K_{\infty}=200-300 \mathrm{MeV}$. This enhanced precision calls for a critical accuracy check at the side of the the theoretical calculations with the goal that the error in the calculated value of $E_{c e n}$ is less than the experimental error.

The basic theory for the microscopic description of different modes of giant resonances is the Hartree-Fock(HF) based random phase approximation (RPA) [5], 6]. Although this approach is conceptually well understood, actual calculations make compromises for reasons of simplicity or numerical expense. One can hardly avoid limitations in the numerical basis and RPA phase space. Furthermore, most of the presently available HF-RPA calculations omit the painful to evaluate pieces of the RPA residual interaction, namely its spin-orbit and/or Coulomb parts. We will call that the self-consistency violation (SCV) in the following. It is obvious that a very accurate calculation within HF-RPA demands a sufficiently complete basis and in particular self-consistency, i.e., using exactly the same pieces in the residual interaction that have been used in the underlying HF calculation. Unfortunately, apart from some fully self-consistent calculations [7, 8, 9, 10, 11], most existing HF-RPA calculations are contaminated by SCV. First assessments on the effects of SCV on the strength functions

of giant resonances were obtained in Refs. [12, 13, 14]. In Ref. [13], results of elaborate studies of the effects of SCV on the constrained energy $\left(E_{c o n}\right)$ and scaling energy $\left(E_{s}\right)$ have been reported only for the ISGMR. It is to be noted that the full self-consistent values of $E_{c o n}$ and $E_{s}$ were obtained using the constrained Hartree-Fock (CHF) approach. It was 
pointed out [13] that the SCV concerning spin-orbit and Coulomb interactions may cause an error in $E_{c o n}$ of the ISGMR which becomes larger than $1 \mathrm{MeV}$, i.e. as large as 5 times the experimental error. This calls for a systematic assessment of the effects of SCV on the excitation energies of other modes as well. Recently in Ref. [9], fully self-consistent calculations of strength function were reported and the shift of the peak of the ISGMR strength function due to SCV has been discussed for ${ }^{120} \mathrm{Sn}$. In Ref. [10], the effect of SCV has been assessed for the IVGDR in ${ }^{16} \mathrm{O}$ using the fully self-consistent approach of small amplitude time dependent Hartree-Fock method. We will continue here with discussing a greater variety of modes, namely the sequence of isoscalar and isovector multi-pole resonances in the range $L=0-3$ and a few examples from low lying collective states.

Before attacking the main task, it is worthwhile to put the various sources of uncertainties into perspective. The HF-RPA method optimizes the modes in the space of one-particleone-hole $(1 p h)$ excitations. Correlations, i.e. $2 p h$ and higher structures are not accounted for explicitly. Such correlations have been very much discussed in the past, for reviews see e.g. 17, 18, 19]. The main effect is a collisional broadening of the strength distributions. This can be accompanied by a certain shift of the resonance peak position which grows with excitation energy and can reach orders of $1 \mathrm{MeV}$ for the rather high lying isovector modes (in the range above $20 \mathrm{MeV}$ ). However, the Skyrme forces employed in nuclear HF and RPA calculations are effective forces which incorporate already a great deal of correlations [20]. This reduces the correlation effects on the peak positions [19, 21]. We adopt the view that the net effect remains of order of a tenth of $\mathrm{MeV}$ for modes with moderate excitation energy around and below $15 \mathrm{MeV}$. A second crucial aspect concerns limitations within the HF-RPA approach itself. There are two major questions: the size of the RPA phase space and the handling of the particle continuum. We take care to use a sufficiently large expansion basis such that peak positions have converged to uncertainties below $0.1 \mathrm{MeV}$. The artificial discretization of the continuum has very little effect on the average peak positions but limits the spectral resolution with which one can reliably compute the strength distributions 22]. We use a large simulation box and properly adapted smoothing width. After all, there remain the effects of SCV to be studied. We demonstrate the accuracy of our fully self-consistent HF based RPA calculations of the strength functions of giant resonances by comparing (i) the RPA results with the corresponding ones of CHF for the case of the ISGMR and (ii) the total energy weighted strengths with the corresponding energy weighted sum rules (EWSR). 


\section{FORMALITIES}

We calculate the strength function,

$$
S(E)=\sum_{j} E_{j}|<0| F_{L}|j>|^{2}
$$

following the fully self-consistent method based on $Q-P$ representation described in Ref. [7, 15]. In Eq. (11), $\mid 0>$ is the RPA ground state and the sum is over all RPA excited states $\mid j>$ with the corresponding excitation energy $E_{j}$. The scattering operator $F_{L}$ is given by

$$
\begin{array}{ll}
F_{L}=\sum_{i} f\left(r_{i}\right) Y_{L 0}(i), & \text { for isoscalar } \\
\left.F_{L}=\frac{Z}{A} \sum_{n} f\left(r_{n}\right)\right) Y_{L 0}(n)-\frac{N}{A} \sum_{p} f(r(p)) Y_{L 0}(p), & \text { for isovector },
\end{array}
$$

with $f(r)=r^{2}, r^{2}$ and $r^{3}$ for monopole, quadrupole and octopole, respectively. For the isovector dipole we have taken, $f(r)=r$, whereas for the isoscalar dipole we adopt the scattering operator $f(r)=r^{3}-\frac{5}{3}<r^{2}>r$ to eliminate the contribution of spurious state mixing [12]. Once we have the strength function, the energy moments can be calculated by,

$$
m_{k}=\int_{0}^{\infty} \omega^{k} S(\omega) d \omega
$$

Then the centroid, constrained and scaling energies of the giant resonance are computed as,

$$
E_{c e n}=\frac{m_{1}}{m_{0}}, \quad E_{c o n}=\sqrt{\frac{m_{1}}{m_{-1}}}, \quad \text { and } \quad E_{s}=\sqrt{\frac{m_{3}}{m_{1}}} .
$$

For the isoscalar $(T=0)$ operator in Eq. (2), the EWSR is given by [2, 5]

$$
m_{1}(L, T=0)=\frac{1}{4 \pi} \frac{\hbar^{2}}{2 m} A\left\langle g_{L}(r)\right\rangle
$$

with

$$
\left\langle g_{L}(r)\right\rangle=\frac{1}{A} \int g_{L}(r) \rho(r) 4 \pi r^{2} d r,
$$

where $\rho(r)$ is the HF ground state matter density distribution and

$$
g_{L}(r)=\left(\frac{d f}{d r}\right)^{2}+L(L+1)\left(\frac{f}{r}\right)^{2}
$$

For the isovector $(T=1)$ operator of Eq. (3), we have,

$$
m_{1}(L, T=1)=\frac{N Z}{A^{2}} m_{1}(L, T=0)\left[1+\kappa-\kappa_{n p}\right]
$$


where $\kappa$ is the enhancement factor due to the momentum dependence of the effective nucleonnucleon interaction, and is given by,

$$
\kappa=\frac{(1 / 2)\left[t_{1}\left(1+x_{1} / 2\right)+t_{2}\left(1+x_{2} / 2\right)\right]}{\left(\hbar^{2} / 2 m\right)\left(4 N Z / A^{2}\right)} \frac{2 \int g_{L}(r) \rho_{p}(r) \rho_{n}(r) 4 \pi r^{2} d r}{\int g_{L}(r) \rho(r) 4 \pi r^{2} d r}
$$

where $t_{i}$ and $x_{i}$ are the parameters of the Skyrme interaction. The correction $\kappa_{n p}$, which arises because of the difference in the profiles of the neutrons and protons density distribution ( ie., since $\rho_{n}(r)-\rho_{p}(r) \neq \frac{N-Z}{A} \rho(r)$ ), is given by,

$$
\kappa_{n p}=\frac{(N-Z)}{A} \frac{A}{N Z} \frac{\int g_{L}(r)\left[Z \rho_{n}(r)-N \rho_{p}(r)\right] 4 \pi r^{2} d r}{\int g_{L}(r) \rho(r) 4 \pi r^{2} d r} .
$$

The fully self-consistent moment $m_{-1}$ for the ISGMR can also be calculated using the constrained Hartree-Fock method [1],

$$
m_{-1}=\left.\frac{1}{4 \pi} \frac{1}{2} \frac{d<r^{2}>_{\lambda}}{d \lambda}\right|_{\lambda=0}=\left.\frac{1}{4 \pi} \frac{1}{2} \frac{d^{2} E_{\lambda}}{d \lambda^{2}}\right|_{\lambda=0}
$$

where the mean square radius $\left\langle r^{2}\right\rangle_{\lambda}$ and the energies $E_{\lambda}$ are obtained using the solution of the constrained Hamiltonian $H_{\lambda}=H-\lambda r^{2}$. The self-consistent energy moment $m_{1}$ can be evaluated [5] using the EWSR of Eqs. (마)-(마) with $f=r^{2}$,

$$
m_{1}=\frac{1}{4 \pi} \frac{\hbar^{2}}{2 m} 4 A<r^{2}>
$$

where $m$ is the mass of the nucleon, $A$ is the mass number and $\left\langle r^{2}\right\rangle$ is the mean square radius calculated using the ground state HF wave function. Thus, using Eqs. (12) and (13), one can get the self-consistent value of $E_{c o n}$ which can also be used to check the accuracy of the RPA calculations (in particular, the completeness of the RPA phase space). For plotting purpose, we employ a Lorentzian smearing of the strength function of Eq. (II) obtaining,

$$
S(E)=\frac{1}{\pi} \sum_{j} \frac{(\Gamma / 2)\left|\left\langle 0\left|F_{L}\right| j\right\rangle\right|^{2}}{\left(E_{j}-E\right)^{2}+(\Gamma / 2)^{2}},
$$

where $\Gamma$ is the smearing parameter taken to be $2 \mathrm{MeV}$ or larger. Note, however, that for the evaluations of the energy moments, Eq. (44), and the energies of various giant resonances, we use a very small value for $\Gamma(<0.2 \mathrm{MeV})$.

Since we shall be investigating the magnitude of errors in the calculation of the observables for giant resonances due to the violation of the self-consistency, it is necessary to pay attention to the accuracy of HF and RPA calculations. We have taken a box of size $30 \mathrm{fm}$. 
This large computational box allows a spectral resolution of the strength functions in the particle continuum of about $0.8 \mathrm{MeV}$ [22] which is by far sufficient for our present investigation. The mesh size is taken to be $0.3 \mathrm{fm}$. The flexible formulation of the RPA [7] in the $Q-P$ representation allows one to include 1 ph excitations up to very high energy, just by taking the detailed 1ph space only for transitions up to few major single particle shell [15]. We have taken the detailed 1 ph space up to 4 particle major shell and checked the convergence of the results by comparing with those obtained with 6 particle major shell. We have adopted the SGII [16] interaction for the HF based RPA calculations and the Slater approximation has been used for the Coulomb exchange term, consistently both in the HF and RPA calculations. For the CHF calculations, we have taken a box of $15 \mathrm{fm}$ with mesh size $0.1 \mathrm{fm}$ and calculated each derivative in Eq. (12) using five point formula with the increment 0.02 in the constraining parameter $\lambda$.

\section{RESULTS AND DISCUSSION}

In order to check the accuracy of our CHF calculations for $m_{-1}$ in ISGMR, we have given the values for ${ }^{40} \mathrm{Ca}$ and ${ }^{208} \mathrm{~Pb}$ in Table 【 calculated from Eq. (12) using the mean square radii, $m_{-1}(r)$, and the energy, $m_{-1}(e)$, methods, for different values of the constraining parameter $\lambda$. For comparison, the values of $m_{-1}$ obtained from RPA calculations Eqs. (II) and (4) are given in the last column. It can be seen that $m_{-1}(r)$ differs from $m_{-1}(e)$ by $0.7 \%$ in the case of $\lambda=0.01$ for ${ }^{40} \mathrm{Ca}$. For $\lambda=0.02$, the deviations are very small $(0.04 \%)$ for both the nuclei. In the following, we will use the value of $\lambda=0.02$ in our calculations. We add that for $\lambda=0.02$ the RPA values of $m_{-1}$, obtained from Eq. (4) using the integration range $0-60 \mathrm{MeV}$, compare very well with the $\mathrm{CHF}$ values (within $0.6 \%$ ). This clearly demonstrates the high accuracy of our CHF and HF-RPA calculations.

A very important necessary condition for a fully self-consistent HF based RPA calculations for giant resonances is to obtain the full EWSR from the calculated RPA strength function. In order to see how this necessary condition is fulfilled in our present calculation, we compare in the Table II, the $m_{1}$ values calculated using the RPA strength functions in Eq. (44), $\left(m_{1}(R P A)\right)$ with the corresponding EWSR obtained from Eqs. (6) and (9) for the nuclei ${ }^{40} \mathrm{Ca},{ }^{90} \mathrm{Zr}$ and ${ }^{208} \mathrm{~Pb}$ for $L=0-3$ and $T=0$ and 1 . It is seen that for all of these

nuclei and for all the modes, the deviations of $m_{1}(R P A)$ from the corresponding EWSR are 
very small (less than $0.3 \%$ ). This once again shows the high accuracy of our HF-RPA calculations. We note that for the SGII interaction we have $\kappa=0.314,0.381,0.314$ and 0.253 and the values of $\kappa_{n p}=0.010,0.000,0.010$ and 0.024 for the isovector $L=0,1,2$ and 3 in ${ }^{208} \mathrm{~Pb}$, respectively. We point out that the correction term $\kappa_{n p}$ in (9) for isovector modes which is usually missing in the literature is not negligible for asymmetric nuclei. As an example, for ${ }^{208} \mathrm{~Pb}, L=3, \kappa_{n p}$ has an effect of $2 \%$ in the calculation of EWSR. The effect of $\kappa_{n p}$ will be more significant for nuclei near the drip lines because of the large difference between the neutron and proton density distributions and also for the large asymmetry $(N-Z) / A$ (see Eq. (11)).

In Table III. we have given the energies $E_{c o n}, E_{s}$ and $E_{c e n}$ of the ISGMR for a host of nuclei ranging from very light to heavy, including some neutron or proton rich nuclei. The second column of Table III indicates the kind of calculation - SC, LS or CO. The symbol SC corresponds to the fully self-consistent calculation, i.e., both the spin-orbit and Coulomb interactions are present in $\mathrm{HF}$ as well as in RPA. The spin-orbit violation (LS) means that the spin-orbit and Coulomb interactions are present in HF but the ph spin-orbit interaction is missing in the RPA calculation. Finally, the Coulomb violation CO means that the spin-orbit and Coulomb interactions are present in HF but the ph Coulomb interaction is dropped out from the RPA calculations. The constrained energies $E_{c o n}$ calculated with the CHF approach using Eqs. (12) and (13) are presented in the third column. The values of $E_{c o n}, E_{s}$ and $E_{c e n}$ of the ISGMR calculated in HF based RPA, are given in the next three columns. The range of integration for calculating the ISGMR energies from the strength function (Eqs. (4) and (5)) is $0-60 \mathrm{MeV}$. Comparison of $E_{c o n}$ obtained from the fully selfconsistent (SC) HF-RPA calculation of the strength function (forth column) with those obtained from the constrained HF method (third column) shows a maximum difference of $0.06 \mathrm{MeV}$. This indicates that the accuracy of our RPA calculation is very good. It is to be noted that the effect of the SCV of LS and CO are similar in $E_{\text {con }}$ and $E_{\text {cen }}$, but a little different in $E_{s}$. The shift of the centroid energy $\delta E_{c e n}$ due to LS or CO violation are listed in the last column. The effects of violation for the spin-orbit (LS) interaction are very small for ${ }^{16} \mathrm{O},{ }^{40,60} \mathrm{Ca}$ and ${ }^{80,110} \mathrm{Zr}$ but robust in other nuclei, particularly for ${ }^{100} \mathrm{Sn}(\sim 1.2 \mathrm{MeV})$ and ${ }^{56} \mathrm{Ni}(\sim 1.8 \mathrm{MeV})$. This can be understood because the effect of the omission of the ph spin-orbit interaction is expected to show up prominently only for the spin unsaturated nuclei like ${ }^{56} \mathrm{Ni}$ and ${ }^{100} \mathrm{Sn}$ here. Hence, for the spin closed ${ }^{16} \mathrm{O},{ }^{40,60} \mathrm{Ca}$ and ${ }^{80,110} \mathrm{Zr}$ nuclei, 
the effects of violation of spin-orbit interaction are very small. On the other hand, the effect of the $\mathrm{CO}$ violation seems to be dependent on the position of the nuclei in the Segre chart.

The energies of the ISGMR are obtained from the ratios of the moments $m_{k}$ in Eq. (5). In Table [V we give for ${ }^{40} \mathrm{Ca}$ and ${ }^{208} \mathrm{~Pb}$ the values of $m_{1}, m_{0}$ and $m_{-1}$ obtained from the fully self-consistent HF-RPA calculations (SC) along with those values obtained from calculations where self-consistency is violated due to the omission of ph spin-orbit (LS) and ph Coulomb (CO) interactions. Comparison of the $m_{1}$ values calculated in fully self-consistent RPA with the EWSR obtained from Eq. (13) (given in column 3) shows that the RPA values are smaller than the corresponding EWSR values by less than $0.3 \%$. The violations of selfconsistency (LS or CO) has little effect on values of $m_{1}(0.04 \%)$ while the effects of SCV are considerably larger in $m_{-1}$ and $m_{0}$ which mainly cause the shift in the giant resonance energies. This shows that obtaining a value of $m_{1}$ in a RPA calculation which is very close to the EWSR does not indicate an accurate and fully self-consistent implementation of HF based RPA theory (see also [12]).

In theoretical study of giant resonances, the evaluation of the strength function $S(E)$ is needed to compute the energy moments (4) and hence the centroid energy, constrained energy, etc. Therefore, it is worth seeing how the strength function as such gets affected by the self consistency violation. In Fig. 1, we display the variation of the $S(E)$ with energy E for isoscalar excitations of different multi-polarities $(L=0-3)$, for the nucleus ${ }^{208} \mathrm{~Pb}$ as a representative case. We have smoothened all the strength functions by a Lorentzian with a smearing parameter $\Gamma=2 \mathrm{MeV}$ (see Eq. (14)) in order to wipe out artefacts from the discretization of the continuum and to provide a smooth curve for better comparison of the effects of SCV in $S(E)$. In the top panel of Fig. 1 we have three curves for the ISGMR $(\mathrm{L}=0, \mathrm{~T}=0)$ — the fully self-consistent ( $\mathrm{SC}$ ) (solid line) result, with violation for ph spin-orbit interaction in RPA (LS) (dashed line) and the result with violation of ph Coulomb interaction in RPA (CO) (open circle). All these three curves have similar single-peaked structure. But it is significant to notice that the violation of LS has pushed the peak to a higher energy by almost by $0.7 \mathrm{MeV}$, whereas the position of the peak of the curve has moved toward the lower energy side by around $0.4 \mathrm{MeV}$ due to the self-consistency violation for the Coulomb interaction. This implies that the self consistency violation may cause an uncertainty in the calculated value of the centroid energy $E_{c e n}$ of ISGMR for ${ }^{208} \mathrm{~Pb}$ which is twice in magnitude compared to the experimental one. This uncertainty, as we have discussed above, would 
impose an error of about $20 \mathrm{MeV}$ in the prediction of the value of $K_{\infty}$. Since the effects of LS and CO violations are opposite to each other, there would be a partial cancellation if one does not take both of the ph LS and CO interactions simultaneously in the RPA calculations. For the isoscalar giant dipole $(\mathrm{L}=1, \mathrm{~T}=0)$ resonance (ISGDR), the effects of neglecting the Coulomb and spin-orbit terms in the ph interaction are similar to those for ISGMR. Violation of LS leads to a push of the peak of $S(E)$ toward a higher energy whereas the violation of $\mathrm{CO}$ pulls the peak to a lower energy. But the magnitude of the shift of the peak is much smaller in comparison with the ISGMR. Specifically, the ph Coulomb interaction shows very little effect. We display similar curves for the higher multi-polarities, $\mathrm{L}=2$ and 3 , in the lower two panels. A shift of the position of the peak of $S(E)$ is observed once we neglect either the ph Coulomb or spin-orbit interactions in the RPA calculation. In both cases of the quadrupole and octopole giant resonances, the effect of the violation of self consistency acts in the same direction which is in contrary to the ISGMR and ISGDR cases. Therefore, the total shift in the peak energy of $S(E)$ will be very significant if one omits both Coulomb and spin-orbit ph interactions in the RPA calculations. Note also the significant shifts in the energies and strengths of the low lying isoscalar $L=1-3$ states due to SCV.

In Fig. 2, we plot the strength functions for the isovector modes for ${ }^{208} \mathrm{~Pb}$ in the same way as we have done in Fig. 1 for the isoscalar mode. Here we have used in the calculations the same parameters as those taken for the isoscalar modes except for the smearing parameter $\Gamma=10 \mathrm{MeV}$ for the isovector giant monopole resonance (IVGMR). It is clear from the figure that in the isovector modes (for all considered multi-polarities) the strength functions are almost insensitive to the self consistency violation due to the omission of the ph spin-orbit interaction in the RPA calculation. On the other hand, the absence of the ph Coulomb interaction in the RPA calculation pushes the strength function significantly toward lower energy and this is most prominent in the isovector dipole mode where the shift is around $0.4 \mathrm{MeV}$.

In Table $\nabla$ we present, for a wide range of nuclei, the self consistent centroid energies $E_{c e n}(S C)$ and their shifts $\delta E_{L S}$ and $\delta E_{C O}$ due to the self-consistency violations for ph spinorbit and Coulomb interactions in the RPA calculations, respectively. We have given results for different multi-polarities ( $L=0,1,2$ and 3 ) for both the isoscalar and the isovector modes. It is evident from the table that for isoscalar modes, the effect of LS or CO violations are most 
prominent for monopole resonance which are almost 2 to 3 times larger than those for the other multi-polarities. Note that if we drop the ph LS and CO interactions simultaneously in the RPA calculations for ${ }^{208} \mathrm{~Pb}$ ISGMR, the shift of the centroid energy is $\delta E_{L S, C O}=-0.30$ $\mathrm{MeV}$ which is comparable to the experimental uncertainty. The effects of the LS and CO self-consistency violations are found to be somewhat smaller for $L=2$ and 3 in comparison with the ISGMR for all of these nuclei. But for the $L=2$ and 3 modes, $\delta E_{L S}$ and $\delta E_{C O}$ are found to be of the same sign. Therefore, their combined effect on the centroid energy is significant. The SCV associated with the Coloumb interaction (in the RPA level) affects $E_{c e n}$ considerably in the dipole and monopole modes of the isovector channel.

Finally, we have checked the effect of SCV on low lying (isoscalar) collective states. Test cases were the lowest $3^{-}$in ${ }^{90} \mathrm{Zr}$ and the lowest $2^{+}$in ${ }^{208} \mathrm{~Pb}$. The low lying states are much more sensitive to the size of the RPA phase space. We have used here an expansion basis up to about $2000 \mathrm{MeV}$ to ensure sufficient convergence. The results are shown in Table VI. The effects are of the same order as found for the giant resonances. However, for these low lying states a mismatch of about $0.5 \mathrm{MeV}$ is large compared to the total excitation energy. A fully consistent calculation is compulsory for studying spectra in that energy range.

\section{CONCLUSION}

In summary, we have carried out highly accurate fully self-consistent Hartree-Fock (HF) based random phase approximation (RPA) calculations for the strength functions of isoscalar and isovector $L=0-3$ modes in a wide range of nuclei. We have quantified very accurately the effects of self-consistency violations in the calculations of the energies of giant resonances of nuclei within the HF based RPA. We have studied the cases of SCV due to the omission of the spin-orbit (LS) or/and Coulomb (CO) ph interactions and mainly focus on their effects on the centroid energy $E_{c e n}$. Here we consider both isoscalar and isovector modes of multi-polarities $L=0-3$. It is found, for the wide range of nuclei considered here, that the effects of violations of self-consistency due to the ph LS or CO interactions are most significant for the ISGMR. For the ISGMR, the absence of the ph LS interaction tends to increase $E_{c e n}$, whereas the violation due to ph CO interaction decreases $E_{c e n}$. For the spin unsaturated nuclei (such as ${ }^{56} \mathrm{Ni}$ and ${ }^{100} \mathrm{Sn}$ ), the shift in $E_{c e n}$ is robust $(\sim 1.5 \mathrm{MeV})$ which is almost 5 times larger than the experimental uncertainty. For other higher multi-polarities, 
the individual effect of the ph LS and CO interactions are somewhat smaller than those for the ISGMR. But for the quadrupole and octopole modes, the LS and CO self-consistency violations both tends to reduce the centroid energy. Hence, the effect of SCV on $E_{c e n}$ in these modes are significant $(0.3-0.6 \mathrm{MeV})$ if one neglects the ph spin-orbit and Coulomb interactions simultaneously in the RPA calculation.

\section{Acknowledgments}

This work was supported in part by the US National Science Foundation under Grant No. PHY-0355200 and the US Department of Energy under the Grant No. DOE-FG0393ER40773.

[1] O. Bohigas, A. M. Lane, and J. Martorell, Phys. Rep. 51, 267 (1979).

[2] E. Lipparini, and S. Stringari, Phys. Lett. B 112, 421 (1982); Phys. Rep. 175, 103 (1989).

[3] D. H. Youngblood, H. L. Clark, and Y. W. Lui, Phys. Rev. C 69, 034315 (2004).

[4] D. H. Youngblood, H. L. Clark, and Y. W. Lui, Phys. Rev. C 69, 054312 (2004).

[5] A. Bohr, and B. Mottelson, Nuclear Structure (Benjamin, London, 1975), Vol. II.

[6] S. Shlomo, and G. F. Bertsch, Nucl. Phys. A243, 507 (1975).

[7] P.-G. Reinhard, Ann. Phys. (Leipzig) 1, 632 (1992).

[8] J. P. Blaizot, J. F. Burger, J. Decharge, and N. Girod, Nucl. Phys. A591, 435 (1995).

[9] J. Terasaki, J. Engel, M. Bender, J. Dobaczewski, W. Nazarewicz, and M. Stoitsov, Phys. Rev. C 71, 034310 (2005).

[10] T. Nakatsukasa, and K. Yabana, Phys. Rev. C 71, 024301 (2005).

[11] J. Piekarewicz, Phys. Rev. C 62, 051304(R) (2000).

[12] B. K. Agrawal, S. Shlomo, and A. I. Sanzhur, Phys. Rev. C 67, 034314 (2003).

[13] B. K. Agrawal and S. Shlomo, Phys. Rev. C 70, 014308 (2004).

[14] G. Colo, and Nguyen Van Giai, Nucl. Phys. A731, 15c (2004).

[15] P.-G. Reinhard, Nucl. Phys. A646, 305c (1999).

[16] Nguyen Van Giai, and H. Sagawa, Phys. Lett. B 106, 379 (1981).

[17] G. F. Bertsch, P. F. Bortignon, and R. A. Broglia, Rev. Mod. Phys. 55, 287 (1983) 
[18] C. Mahaux, P. F. Bortignon, R. A. Broglia, and C. H. Dasso, Phys. Rep. 120, 1 (1985)

[19] P.-G. Reinhard and C. Toepffer, Int. J. Mod. Phys. E 3, 435 (1994)

[20] M. Bender, P.-H. Heenen, and P.-G. Reinhard, Rev. Mod. Phys. 75, 121 (2003)

[21] K. G’utter, K. Wagner, P.-G. Reinhard, and C. Toepffer, Ann. Phys. (N.Y.) 225, 339 (1993)

[22] P.-G. Reinhard, P. D. Stevenson, D. Almehed, J. A. Maruhn, and M. R. Strayer, subm. Phys. Rev. E (2005) 
TABLE I: The values of $m_{-1}$ of ${ }^{40} \mathrm{Ca}$ and ${ }^{208} \mathrm{~Pb}$ for ISGMR obtained from the mean square radii, $m_{-1}(r)$, and energy, $m_{-1}(e)$ methods (Eq. (12) $)$, are presented for different values of the increment of the constraining parameter $\lambda$. For comparison, the RPA values are given in the last column.

\begin{tabular}{|c|ccc|c|}
\hline \hline Nucleus & \multicolumn{3}{|c|}{$\mathrm{CHF}$} & $\mathrm{RPA}$ \\
& $\lambda$ & $m_{-1}(r)$ & $m_{-1}(e)$ & $m_{-1}$ \\
& $\left(\mathrm{fm}^{-2} \mathrm{MeV}\right)$ & $\left(\mathrm{fm}^{4} \mathrm{MeV}^{-1}\right)$ & $\left(\mathrm{fm}^{4} \mathrm{MeV}^{-1}\right)$ & $\left(\mathrm{fm}^{4} \mathrm{MeV}^{-1}\right)$ \\
\hline${ }^{40} \mathrm{Ca}$ & 0.01 & 6.512 & 6.467 & \\
& 0.02 & 6.469 & 6.466 & 6.426 \\
& 0.03 & 6.466 & 6.463 & \\
\hline${ }^{208} \mathrm{~Pb}$ & 0.01 & 230.01 & 230.57 & \\
& 0.02 & 230.57 & 230.50 & 230.83 \\
& 0.03 & 230.27 & 229.89 & \\
\hline
\end{tabular}

TABLE II: Comparison of $m_{1}$ calculated from RPA strength function $\mathrm{S}(\mathrm{E}), m_{1}(R P A)$ (Eq. (4) integrated up to $E_{\max } \sim 100 \mathrm{MeV}$ ), with those obtained from the energy weighted sum rules (EWSR) in Eqs. (6) and (9). The ratio $R=m_{1}(R P A) / E W S R$ indicates that the maximum deviation of $m_{1}(R P A)$ from EWSR in this table is less than $0.3 \%$.

\begin{tabular}{|c|c|cc|cc|cc|}
\hline \hline \multicolumn{2}{|c|}{ Mode } & \multicolumn{2}{|c|}{ Ca40 } & \multicolumn{2}{c|}{ Zr90 } & \multicolumn{2}{c|}{ Pb208 } \\
\hline $\mathrm{L}$ & $\mathrm{T}$ & $\mathrm{EWSR}$ & $\mathrm{R}$ & EWSR & $\mathrm{R}$ & EWSR & $\mathrm{R}$ \\
\hline 0 & 0 & 2889 & 0.9983 & 10505 & 0.9994 & 41872 & 0.9971 \\
& 1 & 896.3 & 0.9992 & 3330 & 0.9994 & 13041 & 0.9998 \\
\hline 1 & 0 & 57253 & 0.9990 & 289907 & 0.9995 & 1823110 & 0.9999 \\
& 1 & 64.62 & 0.9999 & 148.9 & 1.0001 & 337.8 & 0.9999 \\
\hline 2 & 0 & 7222 & 1.0001 & 26262 & 1.0007 & 104681 & 1.0015 \\
& 1 & 2241 & 1.0000 & 8326 & 0.9998 & 32604 & 1.0002 \\
\hline 3 & 0 & 238240 & 0.9996 & 1300645 & 0.9999 & 8584813 & 1.0003 \\
& 1 & 69328 & 0.9994 & 389266 & 0.9996 & 2519881 & 1.0001 \\
\hline
\end{tabular}


TABLE III: The constrained $\left(E_{c o n}\right)$, scaling $\left(E_{s}\right)$ and the centroid energies $\left(E_{c e n}\right)$, in $\mathrm{MeV}$, of the ISGMR of various nuclei are given for fully self-consistent HF-RPA calculations (SC) along with those obtained for the calculations where the self-consistency is violated due to the neglecting of the ph spin-orbit (LS) and Coulomb (CO) interactions in RPA. We adopt the range $\left(\omega_{1}-\omega_{2}\right)=(0-60)$ $\mathrm{MeV}$ for the energy integration. The effects of the SCV on the centroid energies are given in the last column. We have also given in Column 3, the constrained energies obtained from fully selfconsistent constrained Hartree-Fock (CHF) calculations. The Skyrme interaction SGII [16] was used.

\begin{tabular}{|c|c|c|c|c|c|c|}
\hline \multirow[t]{2}{*}{ Nucleus } & \multirow[t]{2}{*}{$\theta_{v}$} & \multirow{2}{*}{$\begin{array}{l}\mathrm{CHF} \\
E_{\text {con }}\end{array}$} & \multicolumn{4}{|c|}{$\mathrm{RPA}$} \\
\hline & & & $E_{\text {con }}$ & $E_{s}$ & $E_{c e n}$ & $\delta E_{c e n}$ \\
\hline \multirow[t]{3}{*}{${ }^{16} \mathrm{O}$} & $\mathrm{SC}$ & 24.025 & 23.985 & 26.846 & 24.584 & \\
\hline & LS & & 23.710 & 26.905 & 24.384 & 0.200 \\
\hline & $\mathrm{CO}$ & & 23.890 & 26.722 & 24.481 & 0.103 \\
\hline \multirow[t]{3}{*}{${ }^{40} \mathrm{Ca}$} & $\mathrm{SC}$ & 21.131 & 21.186 & 22.516 & 21.469 & \\
\hline & $\mathrm{LS}$ & & 21.026 & 22.605 & 21.362 & 0.107 \\
\hline & $\mathrm{CO}$ & & 20.960 & 22.258 & 21.234 & 0.235 \\
\hline \multirow[t]{3}{*}{${ }^{60} \mathrm{Ca}$} & $\mathrm{SC}$ & 16.203 & 16.154 & 18.804 & 16.948 & \\
\hline & LS & & 15.956 & 18.886 & 16.812 & 0.136 \\
\hline & $\mathrm{CO}$ & & 16.093 & 18.660 & 16.865 & 0.083 \\
\hline \multirow[t]{3}{*}{${ }^{56} \mathrm{Ni}$} & $\mathrm{SC}$ & 20.177 & 20.179 & 20.864 & 20.311 & \\
\hline & LS & & 21.853 & 23.056 & 22.075 & -1.764 \\
\hline & $\mathrm{CO}$ & & 19.883 & 20.550 & 20.009 & 0.302 \\
\hline \multirow[t]{3}{*}{${ }^{80} \mathrm{Zr}$} & $\mathrm{SC}$ & 17.841 & 17.873 & 18.629 & 18.050 & \\
\hline & LS & & 17.842 & 18.752 & 18.051 & -0.001 \\
\hline & $\mathrm{CO}$ & & 17.527 & 18.267 & 17.699 & 0.351 \\
\hline \multirow[t]{3}{*}{${ }^{90} \mathrm{Zr}$} & $\mathrm{SC}$ & 17.814 & 17.832 & 18.261 & 17.914 & \\
\hline & LS & & 18.512 & 19.233 & 18.645 & -0.731 \\
\hline & $\mathrm{CO}$ & & 17.532 & 17.944 & 17.609 & 0.305 \\
\hline \multirow[t]{3}{*}{${ }^{110} \mathrm{Zr}$} & $\mathrm{SC}$ & 15.294 & 15.248 & 16.410 & 15.585 & \\
\hline & LS & & 15.207 & 16.596 & 15.584 & 0.001 \\
\hline & $\mathrm{CO}$ & & 15.080 & 16.160 & 15.392 & 0.193 \\
\hline \multirow[t]{3}{*}{${ }^{100} \mathrm{Sn}$} & $\mathrm{SC}$ & 17.156 & 17.207 & 17.645 & 17.282 & \\
\hline & LS & & 18.351 & 19.086 & 18.488 & -1.206 \\
\hline & $\mathrm{CO}$ & & 16.730 & 17.189 & 16.804 & 0.478 \\
\hline \multirow[t]{3}{*}{${ }^{116} \mathrm{Sn}$} & $\mathrm{SC}$ & 16.261 & 16.288 & 16.832 & 16.400 & \\
\hline & LS & & 16.868 & 17.659 & 17.027 & -0.627 \\
\hline & $\mathrm{CO}$ & & 15.961 & 16.478 & 16.062 & 0.338 \\
\hline \multirow[t]{3}{*}{${ }^{144} \mathrm{Sm}$} & $\mathrm{SC}$ & 15.276 & 15.305 & 15.806 & 15.372 & \\
\hline & LS & & 15.991 & 16.740 & 16.100 & -0.728 \\
\hline & $\mathrm{CO}$ & & 14.919 & 15.459 & 14.987 & 0.385 \\
\hline \multirow[t]{3}{*}{${ }^{208} \mathrm{~Pb}$} & $\mathrm{SC}$ & 13.475 & 13.454 & 13.910 & 13.522 & \\
\hline & LS & & 14.080 & 14.767 & 14.196 & -0.674 \\
\hline & $\mathrm{CO}$ & & 13.079 & 13.545 & 13.142 & 0.380 \\
\hline
\end{tabular}


TABLE IV: The values of $m_{k}$ of ${ }^{40} \mathrm{Ca}$ and ${ }^{208} \mathrm{~Pb}$ for ISGMR obtained from fully self-consistent RPA calculations (SC) along with those obtained from the calculations where the self-consistency is violated due to the neglecting of the ph spin-orbit (LS) and Coulomb (CO) interactions in RPA. The EWSR values of $m_{1}$ obtained from Eq. (13) are given in column 3 .

\begin{tabular}{|c|c|c|ccc|}
\hline \hline Nucleus & $\theta_{v}$ & $\begin{array}{c}\text { EWSR } \\
m_{1}(\mathrm{Eq} . \text { (13) }) \\
\left(\mathrm{fm}^{4} \mathrm{MeV}^{-1}\right)\end{array}$ & $\begin{array}{c}m_{1} \\
\left(\mathrm{fm}^{4} \mathrm{MeV}\right)\end{array}$ & $\begin{array}{c}m_{-1} \\
\left(\mathrm{fm}^{4} \mathrm{MeV}^{-1}\right)\end{array}$ & $\begin{array}{c}m_{0} \\
\left(\mathrm{fm}^{4}\right)\end{array}$ \\
\hline${ }^{40} \mathrm{Ca}$ & $\mathrm{SC}$ & 2888.7 & 2884.4 & 6.426 & 134.35 \\
& $\mathrm{LS}$ & & 2884.6 & 6.525 & 135.03 \\
& $\mathrm{CO}$ & & 2885.9 & 6.569 & 135.91 \\
\hline${ }^{208} \mathrm{~Pb}$ & $\mathrm{SC}$ & 41872.5 & 41783.7 & 230.83 & 3090.03 \\
& $\mathrm{LS}$ & & 41782.6 & 210.76 & 2943.37 \\
& $\mathrm{CO}$ & & 41819.6 & 244.49 & 3182.06 \\
\hline
\end{tabular}


TABLE V: Fully self-consistent HF-RPA results of centroid energies $\left(E_{c e n}(S C)\right)$ and their shifts (in $\mathrm{MeV})$ due to self-consistency violations via spin-orbit $\left(\delta E_{L S}=E_{c e n}(S C)-E_{c e n}(L S)\right)$ and Coulomb $\left(\delta E_{C O}=E_{c e n}(S C)-E_{c e n}(C O)\right)$ ph interactions are presented for different modes $(L=0-3$, $T=0,1)$ of some nuclei of experimental interest. The ranges of energies of integrations $\left(\omega_{1}-\omega_{2}\right)$ for the calculations of the centroid energies are given in columns 3 and 7. The Skyrme interaction SGII [16] was used.

\begin{tabular}{|c|c|c|c|c|c|c|c|c|c|}
\hline \multirow[t]{2}{*}{ Nucleus } & \multirow[t]{2}{*}{$\mathrm{L}$} & \multicolumn{4}{|c|}{ Isoscalar } & \multicolumn{4}{|c|}{ Isovector } \\
\hline & & $\omega_{1}-\omega_{2}$ & $E_{\text {cen }}(S C)$ & $\delta E_{L S}$ & $\delta E_{C O}$ & $\omega_{1}-\omega_{2}$ & $E_{c e n}(S C)$ & $\delta E_{L S}$ & $\delta E_{C O}$ \\
\hline \multirow[t]{4}{*}{${ }^{40} \mathrm{Ca}$} & 0 & $5-40$ & 21.31 & 0.12 & 0.21 & $10-60$ & 32.87 & 0.05 & 0.17 \\
\hline & 1 & $22-60$ & 32.60 & -0.09 & 0.08 & $5-40$ & 19.03 & -0.01 & 0.18 \\
\hline & 2 & $8-26$ & 17.16 & 0.24 & 0.07 & $10-60$ & 30.46 & -0.01 & 0.07 \\
\hline & 3 & $22-45$ & 31.48 & 0.30 & 0.02 & $24-65$ & 39.87 & 0.02 & 0.02 \\
\hline \multirow[t]{4}{*}{${ }^{56} \mathrm{Ni}$} & 0 & $5-40$ & 20.20 & -1.68 & 0.30 & $10-60$ & 33.74 & -0.29 & 0.18 \\
\hline & 1 & $22-60$ & 32.78 & -0.54 & 0.10 & $5-40$ & 19.01 & -0.16 & 0.21 \\
\hline & 2 & $8-26$ & 17.52 & 0.32 & 0.12 & $10-60$ & 30.68 & -0.02 & 0.10 \\
\hline & 3 & $22-45$ & 31.17 & 0.55 & 0.03 & $24-65$ & 40.40 & 0.14 & 0.04 \\
\hline \multirow[t]{4}{*}{${ }^{90} \mathrm{Zr}$} & 0 & $5-35$ & 17.89 & -0.68 & 0.30 & $10-55$ & 31.77 & -0.10 & 0.27 \\
\hline & 1 & $18-50$ & 28.88 & -0.33 & 0.09 & $5-35$ & 16.78 & -0.03 & 0.29 \\
\hline & 2 & $8-20$ & 14.64 & 0.15 & 0.17 & $10-50$ & 27.53 & -0.05 & 0.14 \\
\hline & 3 & $20-40$ & 27.31 & 0.29 & 0.05 & $22-60$ & 36.52 & -0.01 & 0.05 \\
\hline \multirow[t]{4}{*}{${ }^{116} \mathrm{Sn}$} & 0 & $5-35$ & 16.38 & -0.59 & 0.34 & $10-50$ & 30.26 & -0.20 & 0.30 \\
\hline & 1 & $18-45$ & 27.39 & -0.33 & 0.10 & $5-35$ & 15.75 & -0.08 & 0.32 \\
\hline & 2 & $8-18$ & 13.60 & 0.14 & 0.19 & $10-45$ & 25.82 & -0.17 & 0.16 \\
\hline & 3 & $18-32$ & 25.37 & 0.40 & 0.05 & $22-55$ & 34.87 & -0.10 & 0.05 \\
\hline \multirow[t]{4}{*}{${ }^{144} \mathrm{Sm}$} & 0 & $5-35$ & 15.34 & -0.69 & 0.39 & $10-50$ & 29.92 & -0.08 & 0.37 \\
\hline & 1 & $18-45$ & 26.42 & -0.33 & 0.13 & $5-35$ & 15.02 & -0.03 & 0.39 \\
\hline & 2 & $8-18$ & 12.97 & 0.11 & 0.28 & $10-45$ & 24.75 & -0.06 & 0.22 \\
\hline & 3 & $18-32$ & 23.95 & 0.14 & 0.04 & $20-50$ & 33.23 & -0.04 & 0.10 \\
\hline \multirow[t]{4}{*}{${ }^{208} \mathrm{~Pb}$} & 0 & $5-30$ & 13.50 & -0.66 & 0.38 & $10-45$ & 27.79 & -0.08 & 0.39 \\
\hline & 1 & $16-40$ & 24.04 & -0.35 & 0.12 & $5-30$ & 13.66 & -0.02 & 0.43 \\
\hline & 2 & $8-16$ & 11.64 & 0.11 & 0.19 & $10-40$ & 22.64 & -0.05 & 0.25 \\
\hline & 3 & $15-28$ & 21.13 & 0.24 & 0.04 & $20-50$ & 30.92 & -0.07 & 0.08 \\
\hline
\end{tabular}


TABLE VI: The effect of SCV for low lying isoscalar states in two selected cases. The Skyrme interaction SGII [16] was used.

\begin{tabular}{|c|c|cccc|}
\hline \hline \multirow{2}{*}{ Nucleus } & \multirow{2}{*}{$\mathrm{L}$} & \multicolumn{4}{|c|}{ energies $[\mathrm{MeV}]$} \\
& & $\mathrm{SC}$ & $\mathrm{LS}$ & $\mathrm{CO}$ & LS-CO \\
\hline${ }^{90} \mathrm{Zr}$ & 3 & 2.10 & 2.57 & 1.95 & 2.47 \\
\hline${ }^{208} \mathrm{~Pb}$ & 2 & 4.48 & 5.06 & 4.38 & 4.99 \\
\hline
\end{tabular}



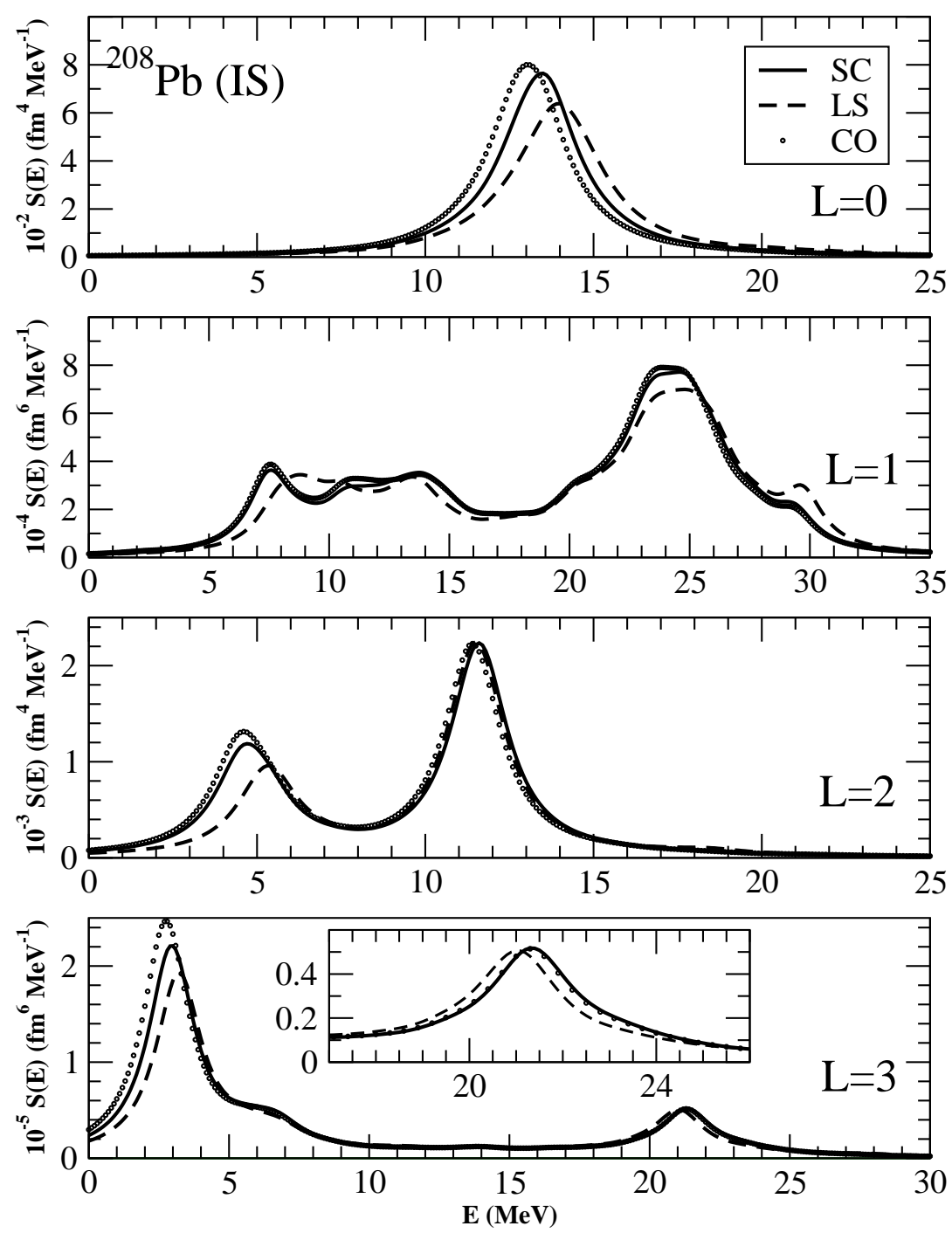

FIG. 1: HF-RPA results for isoscalar strength functions of ${ }^{208} \mathrm{~Pb}$ for $L=0-3$ multi-polarities are displayed. SC (full line) corresponds to the fully self-consistent calculation where LS (dashed line) and CO (open circle) represent the calculations without the ph spin-orbit and Coulomb interactions in the RPA, respectively. A magnified giant resonance is shown in the inset (lowest panel) for $L=3$. The Skyrme interaction SGII [16] was used. 

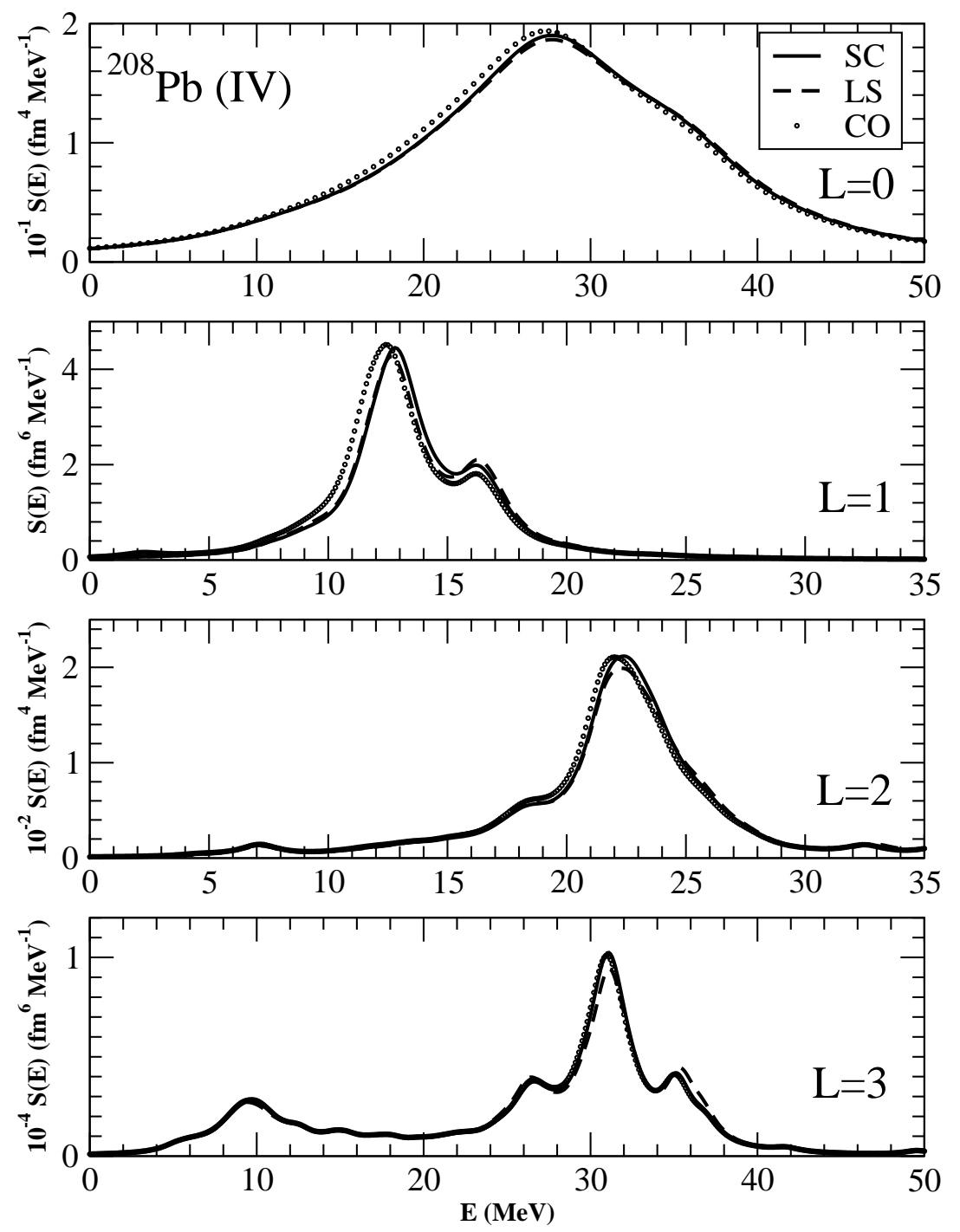

FIG. 2: Same as Fig 1 but for isovector strength functions. 